\title{
Inhibitory Effect of Dietary Curcumin on 1, 2-Dimethylhydrazine-Induced Colon Preneoplasia in Irradiated Rats
}

\author{
Manar Nabih Hafez \\ National Centre for Radiation Research and Technology, Atomic Energy Authority
}

\begin{abstract}
Introduction: Prevention of cancer remains a primary need and new chemopreventive agents must be developed for this purpose. It is well known that the radiotherapy is associated with severe side effects and offers no firm expectation for a cure. Thus, there is a constant need for the investigation of other potentially useful options. One of the widely sought approaches is cancer chemoprevention that uses natural agents. Curcumin, a natural plant product, has been shown to have anti-inflammatory and antioxidant properties and which, thus, may be a potential anticancer drug.

Material and Methods: The inhibitory effects of dietary curcumin $(0.6 \%$ in diet) on colonic precancerous lesions in rats treated with 1,2-dimethylhydrazine, (DMH, $20 \mathrm{mg} / \mathrm{kg}$, s.c. once weekly for 6 weeks) or exposed to $\gamma$-radiation $(6.5 \mathrm{~Gy})$ alone or in combination were evaluated histologically and histochemically.

Results: The results showed clear features of colonic dysplasia with DMH treatment. The dysplastic crypts exhibited marked hyperchromatism, loss of polarity besides nuclear and cellular stratification. Whereas, $\boldsymbol{\gamma}$-irradiation revealed marked crypt dilatation accompanied by disorganized crypt structure and alteration of surface epithelium. The results also demonstrate an apparent synergy between the radiation and the chemical carcinogen in the induction of augmentation of mucosal abnormalities accompanied with increased total DNA content and cell proliferation. Consistent with these observations, changes in the type of mucous secretion with predominance of sialomucins were observed in the areas of mild to moderate dysplasia whilst the surrounding normal epithelium produced sulphated material. Mucus depletion was a common feature in areas of severe dysplasia.

Addition of curcumin to the diet remarkably reduced the amount and severity of dysplastic lesions with DMH-induced colon preneoplasia without appreciable toxicity. Moreover, curcumin could prevent or improve the disruption of colonic architecture caused by $\gamma$ irradiation. Such improvement was also investigated but to a lesser extent with the combined radiation and $\mathrm{DMH}$.

Conclusion: These results indicated that curcumin is potent in inhibiting the preneoplastic lesions and other cellular events relevant to colon and was, therefore, of value as a safe chemotherapeutic agent.
\end{abstract}

\section{Introduction}

Colon cancer is one of the most common cancer in developed countries and is considered to be one of the major causes of cancer-related death (Manju et al., 2005). Virtually every major form of human cancer can be reproduced in experimental animals by exposure to specific chemical carcinogens (Yuspa and Poirier, 1988).1, 2-
Dimethylhydrazine (DMH) has been shown to induce colonic carcinomas in rats and mice with marked organ specificity (Sitohy and El-Salhy, 2001). Also, Maskens (1976) reported that DMH treatment enhances epithelial cell proliferation in the rat colon .

Deschner and Long (1977) found that whatever alterations are induced by $\mathrm{DMH}$ 


\section{Manar Nabih Hafez}

early in treatment, the changes are sufficient for induction of tumours when adequate time for tumour development is given.

The clinical application of combined modality therapy involving radiation and chemotherapeutic agents is growing in importance. Unfortunately, radiation and a number of the more common chemotherapeutic agents, particularly alkylating agents, are potential carcinogens. The increasingly successful application of combined modalities for treatment of malignancies has led to growing awareness of the risk of second malignancies (Penn, 1988). Although there are many reports of the carcinogenicity of various single agents, the potential for synergism between carcinogens and radiation does not appear to have been widely studied (Reif, 1984). This is surprising because these agents are used together therapeutically and potent carcinogens and radiation are present together in the environment. Thus in these situations, there exists the potential for synergism in the induction of tumours.

Several epidemiological and experimental studies suggest a relationship between colon cancer risk and dietary factors. Many compounds in food, including some nutrients have been reported to promote colon cancer, whereas some nutrients and many phytochemicals have been shown to have colon cancerpreventive properties (Key et al., 2002).

It is noteworthy that the use of medicinal plants or their crude extracts in the prevention and / or treatment of several chronic diseases has been traditionally practiced in various different ethnic societies worldwide; however, their use in modern medicine suffers from lack of scientific evidence. Only very few medicinal plants have attracted the interest of scientists and one such plant is Curcuma Longa Linn. The powdered rhizome of this plant, turmeric, has been extensively used for imparting colour and flavor to foods and also for the treatment of variety of inflammatory conditions and chronic diseases (Ammon and Wahl, 1991). Curcumin (diferuloylmethane), a phenolic compound which has been identified as the major yellow pigment in turmeric, possesses both anti-inflammatory and antioxidant properties (Ukil et al., 2003 and Holt et al., 2005).

In the earlier studies, it has been found that dietary administration of $2 \%$ curcumin in the diet inhibited the azoxymethane (AOM)-induced small and large intestinal tumours in mice (Huang et al., 1993). Also, Rao et al. (1993) showed that $2000 \mathrm{ppm}$ curcumin in the diet significantly suppressed the AOM-induced colonic aberrant crypt foci formation, which are early preneoplastic lesions in male rats. It has been demonstrated that topical application of curcumin inhibits benzo $(a)$ pyrene-induced DNA adduct formation and development of skin tumours as well as TPA3 (12-O-tetradecanoylphorbol-13-acetate)-induced epidermal DNA synthesis and tumour promotion in mouse skin (Huang et al., 1997). Curcumin (480 mg orally 3 times a day) appears to reduce the number and size of ileal and rectal adenomas in patients with familial adenomatous polyposis without appreciable toxicity (Cruz-Correa et al., 2006).

The use of conventional biopsy and histology is of limited value in interpreting precancerous changes in the bowel; for example, the presence of inflammatory cells may interfere with the diagnosis of dysplasia in ulcerative colitis (Morson, 1972). Conventional histology will confirm advanced and even some early neoplastic changes, but it is a less reliable method of detecting subtle preneoplastic changes especially at the nuclear level. It has been suggested that an increase in the sialomucin content of colonic mucosa is an indicator of premalignancy (Filipe, 1975) although hypersecretion of sialomucin has also been demonstrated in non-neoplastic diseases of 


\section{Manar Nabih Hafez}

the colon (Franzin et al., 1983). Matthews and Cooke (1986) reported that the amount of DNA in an individual cell reflects its stage in the cell cycle so proliferative changes in colonic mucosa may be reflected by an alteration in the mean DNA content of the crypt cells.

It was, therefore, of interest to study the inhibitory effects of dietary curcumin on DMH-induced colonic preneoplastic lesions in irradiated rats. Histopathological study as well as mucous secretion and DNA content have been investigated in the precancerous colonic mucosa in an attempt to determine whether pre-neoplasia can be recognized.

\section{Material and Methods}

\section{Animals and Diets.}

Male albino rats (supplied by the breeding unit of Holding Company for Biological Product and Vaccines) with an initial weight of $140 \mathrm{~g}$ were used in all the experiments. Rats were housed in plastic cages with filter tops (five per cage) under controlled conditions of light, humidity and temperature at the Animal House Lab., National Centre for Radiation Research and Technology, Cairo. All animals were allowed standard laboratory diet and water ad libitum and acclimatized in our animal facility for two weeks prior to use in any experimental protocol.

The experimental diet was prepared by adding curcumin at $0.6 \%$ level to the control diet as described by Kawamori et al. (1999). Curcumin (> 98\% of diferuloylmethane) was purchased from Sigma Chemical Co., St Louis, MO. The incorporation of curcumin into the control diet was done with V-blender after curcumin was premixed with a small quantity of diet in a food mixer to ensure its uniform distribution. All feeds were pelleted to avoid stratification and to assure uniform feed and curcumin intake in the treated animals. The control and experimental diets were prepared weekly and stored in a cold room. Animals had access to food and water at all times and food cups were replenished with fresh diet three times weekly.

\section{Materials.}

1,2-Dimethylhydrazine-2HCl (DMH) were purchased from Sigma Chemical Co., St Louis, Mo. DMH was dissolved in a 1.5 $\mathrm{mM}$ EDTA solution and adjusted to $\mathrm{pH} 6.5$ with $1 \% \quad \mathrm{NaOH}$ before s.c. injection (Heitman et al., 1982).

\section{Irradiation Procedure:}

Whole body gamma-irradiation was performed using an indoor shielded ${ }^{137} \mathrm{Cs}$ irradiator type Gamma cell-40 installed in the National Centre for Radiation Research and Technology, Atomic Energy Authority, Cairo, Egypt, emitting a dose of irradiation at the rate of $0.61 \mathrm{~Gy} / \mathrm{min}$.

\section{Experimental Design:}

One hundred and twenty male albino rats were divided randomly into eight groups. The $1^{\text {st }}$ group $(\mathrm{n}=10)$ served as controls. The $2^{\text {nd }}$ group $(n=20)$ was given 6 weekly s.c. injections of DMH at a dosage of $20 \mathrm{mg} / \mathrm{kg}$ b.wt. Injections were given at weekly intervals, because it has been shown to be the time needed for the colonic mucosa to recover following the acute toxic effects of a single injection of DMH (Maskens, 1979). The $3^{\text {rd }}$ group $(n=15)$ was exposed to sublethal dose of $\gamma$-radiation at a single dose level of $6.5 \mathrm{~Gy}$. As reported by Yarmonenko (1988), the $6.5 \mathrm{~Gy}$ is a sublethal dose for rats. It has been reported that this dose was low enough to initiate a proliferative response in the crypt cells without killing the animals. The $4^{\text {th }}$ group $(n=20)$ received combined radiation-DMH treatment. The rats were given $6.5 \mathrm{~Gy}$ of whole body irradiation one day before the beginning of the first week of $\mathrm{DMH}$ 


\section{Manar Nabih Hafez}

injection, so that irradiation effects on initial DMH-induced changes in the cell could be tested. It is worthy to mention that rats in groups 1-4 were fed standard Lab diet. The $5^{\text {th }}$ group $(\mathrm{n}=10)$ was given $0.6 \%$ curcumin in control diet for 12 weeks. The $6^{\text {th }}-8^{\text {th }}$ groups $(\mathrm{n}=45)$ were given curcumin diet and water ad libitum from two weeks before the first injection of DMH or irradiation and continuing until the end of experiment.

\section{Screening test of DMH administration:}

According to Kanna et al.(2003), treatment with $20 \mathrm{mg} / \mathrm{kg}$ 1,2-DMH for 6 weeks resulted in preneoplastic lesions in rat colon. In this study, several histopathological examinations were performed to monitor premalignant changes in the colon before the appearance of frank carcinoma in the animals. The first group was sacrificed immediately after the last week of DMH injection and subsequent groups were killed at 10 and 14 weeks after the initial injection. The results revealed the development of colonic preneoplastic changes at 10 weeks and remained unchanged until the end of 14 weeks. Therefore in the present experiment, animals were killed under deep ether anaesthesia at four weeks after the last dose of DMH injection as well as at 1, $3 \&$ 7 days post-irradiation.

After laparotomy, the colones were resected and opened longitudinally and the contents were flushed with normal saline. A $1 \mathrm{~cm}$ length of colonic specimens were fixed in $10 \%$ neutral buffered formol saline. All of the fixed samples were routinely processed and embedded in paraffin wax. Sections of $5 \mu \mathrm{m}$ thickness were cut and stained with haematoxylin and eosin (Drury and Wallington, 1980) for histological assessment. other sections were stained by the following histochemical techniques: high-iron-diamine-Alcian blue (HID-AB) for the visualization of acid mucins (sialoand sulphomucins) (Spicer, 1965), Alcian blue-periodic acid-Schiff (AB-PAS), for the identification of acid and neutral mucosubstances (Mowry, 1956) and Feulgen technique for DNA (Troyer, 1980).

The optical densities (O.D.) of the acid mucins and DNA stained sections were measured by image analyzer. The numerical values of O.D. were analyzed statistically using Student's $t$-test.

\section{Results}

\section{I- Histopathological Results}

Controls: Normal colon mucosa is illustrated in figures $(1 \mathrm{~A} \& \mathrm{~B})$.

DMH. treated groups: As opposed to the crypts of the normal colon mucosa that have a test-tube shape and parallel lateral walls, some crypts from the abnormal colon mucosa (Figs. 2A $-\mathrm{D}$ ) showed dilated lumen. Some crypts were hyperdistended with mucosubstances, while others showed a distinct overcrowding of cells with little or no mucus production. Overcrowded crypts were found to contain focal areas of nuclear and cellular stratification (atypia). Various degrees of dysplastic lesions were present, where the crypts are not usually longer and may be dilated and the goblet cells are globular. Moreover, distortion of the crypts contours, nuclear hyperchromatism and moderate pseudostratification were seen. There was also a sort of atypism in the same focus of dysplasia or even in the same crypt, i.e. the crypts showing marked hyperchromatism, loss of nuclear polarity and epithelial cell proliferation. The occasional mild fibrosis in the lamina propria was also demonstrated. Nuclear anomalies could be seen in the colonic epithelium comprising nuclear fragments, large pyknotic nuclei and darkly stained bodies which were often contained within vacuoles.

Irradiated group: Colonic tissue were obtained from irradiated rats at $1,3 \&$ 7 days. At one day after exposure (Fig. 3), there was some evidence of crypt disorgan- 


\section{Manar Nabih Hafez}

ization. The crypts are longer, the lumen may be dilated and the goblet cells are numerous and distended with mucus. Overcrowded crypts with some nuclear atypia were observed. Occasionally, continuity of the crypt lining (eosinophilic) cells with the surface epithelium could be demonstrated. Irradiation also induced sloughing of cells and an increase in pyknotic nuclei along the lower halves of the crypts.

The most marked effects were at three days. The major observations were marked crypt dilatation accompanied by disorganized crypt structure and alteration of surface epithelium. Some crypts showed breakdown of the continuity with some nuclear changes (Fig. 4). Seven days postexposure, there was an apparent amelioration of colon mucosa structure. Surface mucosa was near normal although some hyperplasia, and little evidence of crypt dilatation were observed (Data not shown). At all times during the experiment, lamina propria appeared infiltrated by mixed inflammatory cells.

Irradiated- DMH. treated group: The combination of radiation with DMH resulted in an augmentation of the number and severity of the colonic lesions than by either agent; where disorganization of crypt structure was progressive (Figs. 5A-D). The dysplastic nature of the epithelium and focal nuclear atypia were apparent. Irregularity of size, shape and staining of the nuclei was seen. Foci of cell proliferation, loss of polarity and marked nuclear hyperchromatism had been observed. In the area of severe dysplasia, goblet cells were rare and thus mucus production was very much reduced. However, in the area of hyperplasia, hyperdistended crypts contained abundant mucus. Some crypts were heavily damaged, others were depleted and replaced by severe inflammatory cellular infiltrates. Mucosal surface was also denuded.

Curcumin fed groups: Experimental diets containing curcumin did not result in any colon structural alterations during the experimental investigation. The results also indicated that administration of curcumin in the diet before, during and after DMH treatment resulted in an apparent amelioration of colonic crypt structure, where most crypts appeared almost normal (Figs. 6A \& B). However, some crypts exhibited various degrees of colonic injury, where crypt dilatation, diffuse cellular infiltrates and extravasation of RBCs were still noticed besides few deformed crypts with foci of hyperplasia.

Moreover, curcumin could produce marked protection against the deleterious responses of irradiation. The mucosa was obviously regenerated with no inflammatory reaction (Fig. 7). The majority of crypts seemed to be normal, whereas others showed focal hyperplasia and some nuclear changes in their lining cells. In addition, administration of dietary curcumin to rats during the combined radiation and $\mathrm{DMH}$ treatments showed that mucosa was not markedly disrupted (Fig. 8) as compared to those fed the control diet only. The colonic mucosa contained crypts lined with slightly crowded columnar cells and decreased numbers of goblet cells. Alterations of surface epithelium (focal breakdown of continuity and hyperplasia) have been demonstrated in some areas of crypts, whereas in other areas, many crypts were fully regenerated. 


\section{Manar Nabih Hafez}
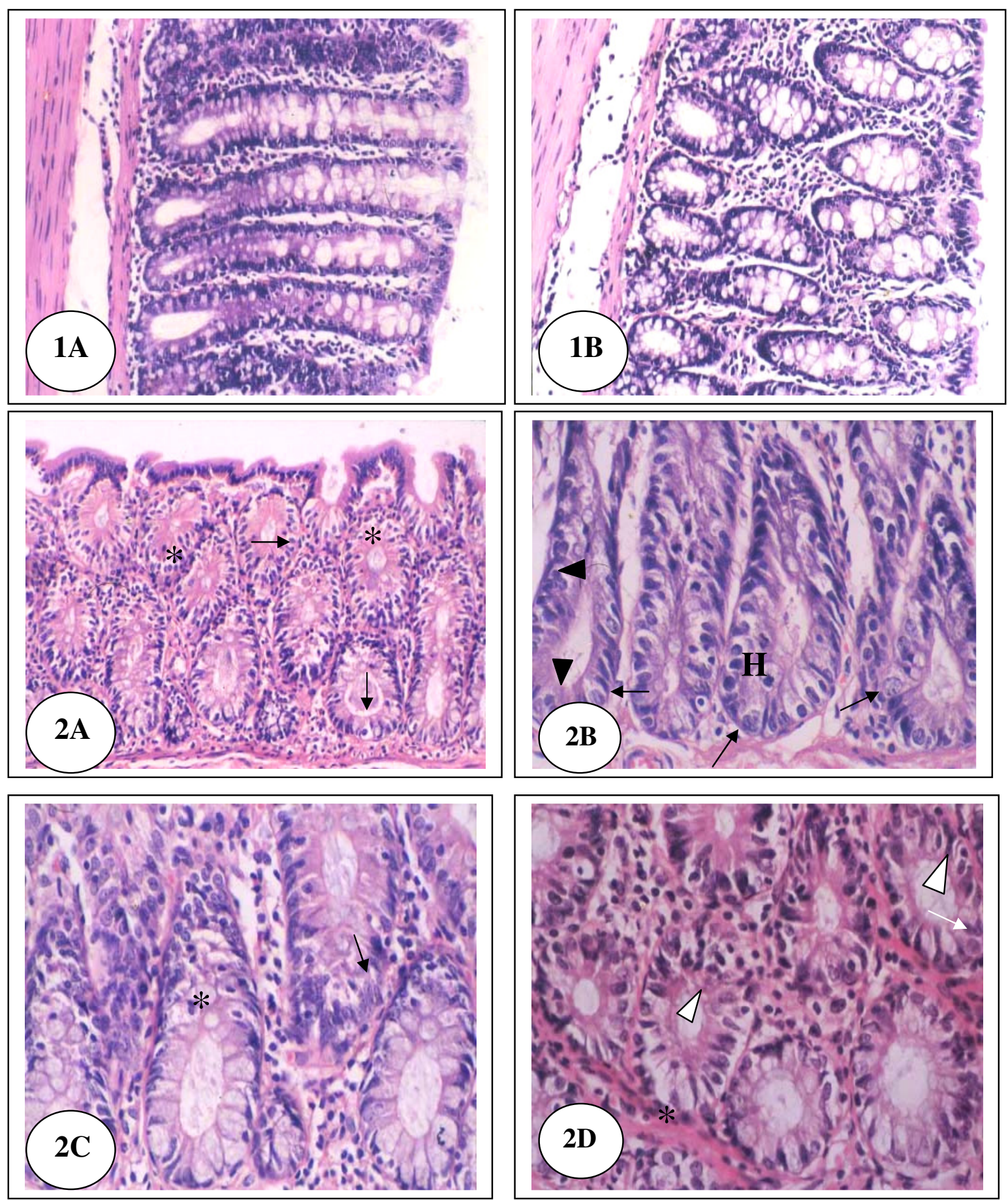

Figs. (1 A \& B): Sections in the colonic mucosa of control rats showing:

(A): Straight crypts lined with abundant goblet cells intermixed with unremarkable columnar cells. Lamina propria contains scattered lymphocytes and plasma cells (H \& E, X 200).

(B): Control crypts lined with mucus secreting goblet cells (H \& E, X 200).

Figs. (2 A - D): Sections in the clonic mucosa of rats treated with DMH showing:

(A): Hyperplastic glands with some dysplastic nuclear changes in the lining cells. The crypts exhibit hyperchromatic nuclei $(\rightarrow)$, decreased numbers of goblet cells as well as nuclear and cellular stratification (*) (H \& E, X 200).

(B): Distorted crypts with hyperdistended lining cells. Note large $(\rightarrow)$ and hyperchromatic nuclei $(\mathrm{H})$, loss of polarity ( ) and cellular stratification (H \& E, X 400).

(C): Dilated crypts with moderate pseudostratification (*). Most of the nuclei are confined to the basal half of the cells. Note signs of nuclear anomalies (fragmented nuclei) $(\rightarrow)$ and an inflammatory reaction in the lamina propria (H \& E, X 400).

(D): Atypical crypts with nuclear hyperchromatism $(\rightarrow)$ and loss of polarity $(\rightarrow)$. Note that some crypts are lined with tall colummar cells. Collagen deposition is observed in the lamina propria (*) (H \& E, X 400). 